\title{
An integrative theory for cancer (Review)
}

\author{
GUOPEI LUO ${ }^{1,2}$ and NA LIU ${ }^{3}$ \\ ${ }^{1}$ Department of Pancreatic Surgery, Fudan University Shanghai Cancer Center; ${ }^{2}$ Department of Oncology, \\ Shanghai Medical College, Fudan University, Shanghai 200032; ${ }^{3}$ Department of Psychiatry, \\ Shanghai Mental Health Center, Shanghai Jiao Tong University School of Medicine, Shanghai 200030, P.R. China
}

Received September 6, 2018; Accepted November 21, 2018

DOI: 10.3892/ijmm.2018.4004

\begin{abstract}
In the integrative theory, chronic irritations induce tumors with genetic alterations and rapid proliferative ability. Tumor cells reprogram the metabolism and employ aerobic glycolysis to sustain rapid growth. The host provides both the nutrients and exhaust system to support tumor growth via the tumor microenvironment. Under certain conditions, such as aging, diabetes, obesity and a high-fat diet, the exhaust system is impaired, triggering a metabolic imbalance between the tumor and host. This is similar to a problematic car with an advanced motor with an out-of-date exhaust system. The metabolic imbalance causes a metabolic catastrophe, making tumor cells reside in a dismal environment and forcing them to invade, metastasize and undergo necrosis. Tumor necrosis, particularly in metastases, leads to non-specific systemic inflammation, which is the major cause of cancer-related mortality. On the whole, the integrative theory views cancer in an integrative manner and proposes that both genetic alterations and tumor-host interaction as regards metabolism and immunology determine the destiny of the tumor and host. Although cancer is a genetic disease, tumor biology is basically the nature of the host.
\end{abstract}

\section{Contents}

1. Introduction

2. Tumor metabolism is reprogrammed for rapid growth

3. A host with an impaired metabolism generates metabolic catastrophe

Correspondence to: Dr Guopei Luo, Department of Pancreatic Surgery, Fudan University Shanghai Cancer Center, 270 Dong'An Road, Xuhui, Shanghai 200032, P.R. China

E-mail: luoguopei@hotmail.com

Dr Na Liu, Department of Psychiatry, Shanghai Mental Health Center, Shanghai Jiao Tong University School of Medicine, 600 Wan Ping Nan Road, Xuhui, Shanghai 200030, P.R. China

E-mail: lnmilk-1982@126.com

Key words: metabolic reprogramming, genetic mutation, chronic irritation, systemic inflammation, immunologic response, cancer development, host
4. Metabolic imbalance induces cancer formation

5. Metabolic imbalance forces the tumor to invade and metastasize

6. Metabolic imbalance stimulates cachexia

7. Metabolic imbalance provokes systemic non-specific inflammation via tumor necrosis

8. Systemic non-specific inflammation causes cancer-related mortality

9. Hexosamine biosynthetic pathway (HBP) pathway regulates metabolism and cancer evolvement

10. The integrative theory applied in specific conditions

11. Therapeutic potential

12 Conclusion

\section{Introduction}

As one of the leading causes of mortality, cancer constitutes a major health burden worldwide (1-4). Its incidence has maintained an upward trend in recent decades due to an increasing prevalence of established risk factors, such as air pollution, diabetes, being overweight, physical inactivity, smoking, lifestyle changes and notably, the aging of the population $(2,3)$. Although major advances have been made in cancer research, there is still much uncertainty, both among the public and among researchers, as regards certain fundamental questions. For example, while cancer is clearly a genetic disease, it remains unclear as to why only a minority of individuals with certain oncogenic mutations (e.g., KRAS mutations) develop cancers. In addition, while smoking is a well-known risk factor for the development of lung cancer, it is unclear as to why the majority of smokers are exempt from this malignancy. More importantly, while $>90 \%$ of cancer-related mortality is attributed to metastases, it remains unclear as to why specific organ failure is rarely observed at the time of a patient's death. In this review, we propose the integrative theory, in which both oncogenic mutations and tumor-host interaction determine the fate of the cancer and host (Fig. 1). Moreover, cancer is clearly a genetic disease, while tumor biology is basically the nature of the host.

\section{Tumor metabolism is reprogrammed for rapid growth}

Similar to normal cells, cancer cells require adenosine triphosphate (ATP) and nutrients to proliferate. However, they notably differ from normal cells due to their dependence on glycolysis 
even under aerobic conditions, which is known as the Warburg effect (5-7). This phenomenon has been well demonstrated by the wide use of $18 \mathrm{~F}$-fluorodeoxyglucose-positron emission tomography (FDG-PET) for tumor detection in clinical practice, which shows an increased glucose uptake in tumors by the glycolysis process (7). Compared with tricarboxylic acid (TCA) releasing 36 ATPs per cycle, glycolysis is rapid, but inefficient, producing only 2 ATPs each cycle (8).

The Warburg effect may be explained by the increased biosynthetic demand of cancer cells, as glycolysis can provide metabolic intermediates for cellular biosynthesis (Fig. 2) (9-11). Human bodies are exposed to various types of chronic irritations, including air pollution, smoking, infection, a high-fat and high-protein diet, alcohol consumption and radiation. Based on the evolution theory, cells with the potential to rapidly self-renew (stem and progenitor cells) are susceptible to irritations and remodel to rapid growth (12). Long-term or strong irritations may cause genetic mutations in normal cells (13). These genetic mutations, alongside with inherited genetic alterations, can induce cancer with uncontrolled rapid growth, which is the leading feature of malignancy $(12,14,15)$. Tumor cells can obtain anabolic substrates (e.g., 5-ribose phosphate) to support uncontrolled growth through aerobic glycolysis and the pentose phosphate pathway (PPP) (16). The major hallmarks of cancer cells universally center on maintaining uncontrolled rapid growth, including sustaining proliferative signaling, evading growth suppressors, enabling replicative immortality, inducing angiogenesis, genomic instability and mutations, and resisting cell death (17). Indeed, normal cells with the ability of rapid division and growth (e.g., embryonic cells) also harbor active glycolysis (18). Certain metabolites produced during embryo formation are increased in malignant tumors [e.g., carcinoembryonic antigen (CEA) in colorectal cancer] (19). Therefore, metabolism is reprogrammed for the rapid growth of cancer cells.

\section{A host with an impaired metabolism generates metabolic catastrophe}

The host provides both nutrients and an exhaust system to support tumor growth via the tumor microenvironment. To cope with the reprogrammed metabolism in cancer cells, an intact metabolic system of the host is essential to maintain tumor growth. However, the metabolic capacity of the host can be impaired under certain conditions, such as aging, diabetes, obesity, a high-fat and high-protein diet, and physical inactivity. The reprogrammed metabolism in cancer cells and the impaired metabolic capacities in the host create a metabolic catastrophe, which inhibits easy cancer growth and accelerates its progression (20-22). This point has been supported by previous studies, which have demonstrated that cancer patients who are frail with metabolic capacities have a poorer prognosis than cancer patients with an intact metabolism (23-25), indicating that the combined effect of cancer and systematic disease (e.g., diabetes) is more detrimental than either factor alone $(1+1>2)$. Several conditions of impaired metabolic capacity are interpreted below.

Aging. Aging has been considered the most potent carcinogen and it has aided the increased cancer incidence, as a growing number of the population nowadays live to an old age worldwide $(26,27)$. To a certain extent, cancer is largely an aging disease and it is similar to other age-related disorders, despite differential manifestations (28). In an experiment using animal models with TP53 mutation, a shorter latency of tumor development occurs when the gene is mutated in older animals (29). The accumulation of genetic mutations has been considered the major cause of cancer as a carcinogen, resulting in an assumption that the probability of cancer development is positively associated with age. However, this is contrary to clinical observations, in which the incidence of different types of cancer occurs at different ages (mesenchymal or hematopoietic cancers at a young age and epithelial carcinomas from ages between 40 to 80 years) (30). Moreover, cancer morbidity and mortality even decline in the oldest age group, including in centenarians (31). In fact, aging is featured by a progressive decline in the function of multiple tissues, with an increase in hyperplasia in renewable tissues (28). Normal cells with a strong renewal capacity are vulnerable to various types of stresses and have high probability of both stress-induced and spontaneous mutations $(32,33)$. Additionally, aging is usually accompanied by an increasing chronic inflammation, which can stimulate cancer progression (34). Importantly, the metabolic capacity of the host is undoubtedly impaired as aging, creating a harsh and infertile milieu, forces the tumor to invade and colonize (30). Therefore, the association between cancer and aging is mainly due to the combined effects of genetic accumulation, immunologic degeneration and more importantly, age-related degeneration in the metabolic systems $(13,30-33)$.

Diabetes. The association between cancers and diabetes has been noted for almost 200 years (35). Diabetes is a risk factor for various types of cancer, such as breast, pancreatic and colorectal cancers $(23,24,36,37)$. Importantly, diabetes is associated with the poorer worse survival of cancer patients $(25,38-41)$. A potential mechanism is that the metabolic capacity of diabetic patients is remodeled to hyperinsulinemia, insulin resistance and insulin-like growth factor (IGF) signaling, causing an enhanced HBP activation and O-GlcNAc synthesis (42-44). The metabolism of cancer cells is characterized by aerobic glycolysis, leading to a strengthened HBP activation and O-GlcNAc synthesis $(8,45)$. For patients suffering from both cancer and diabetes, the HBP pathway is magnified to produce $\mathrm{O}-\mathrm{GlcNAc}$, which plays a critical role in the oncogenic process via post-translational modification (45). This could be demonstrated by the clinical observation that patients with both cancer and diabetes presents dismal prognosis $(1+1>2)(25)$. The association between diabetes and pancreatic cancer is an example of this proposition. It has been shown that only $14 \%$ of patients with pancreatic cancer have normal fasting glucose values (46) and the association is more evident in cases with worsening or new-onset diabetes $(47,48)$. O-GlcNAc is the precursor of carbohydrate antigen 19-9 (CA19-9), an important biomarker of pancreatic cancer (49). Patients with normal levels of CA19-9 have been shown to have a surprisingly improved survival than patients with CA19-9 overproduction (50). Meanwhile, serum CA19-9 levels are often elevated in diabetic patients and correlate with poor glycemic control $(51,52)$. For diabetic patients with pancreatic cancer, the synergistic effect 


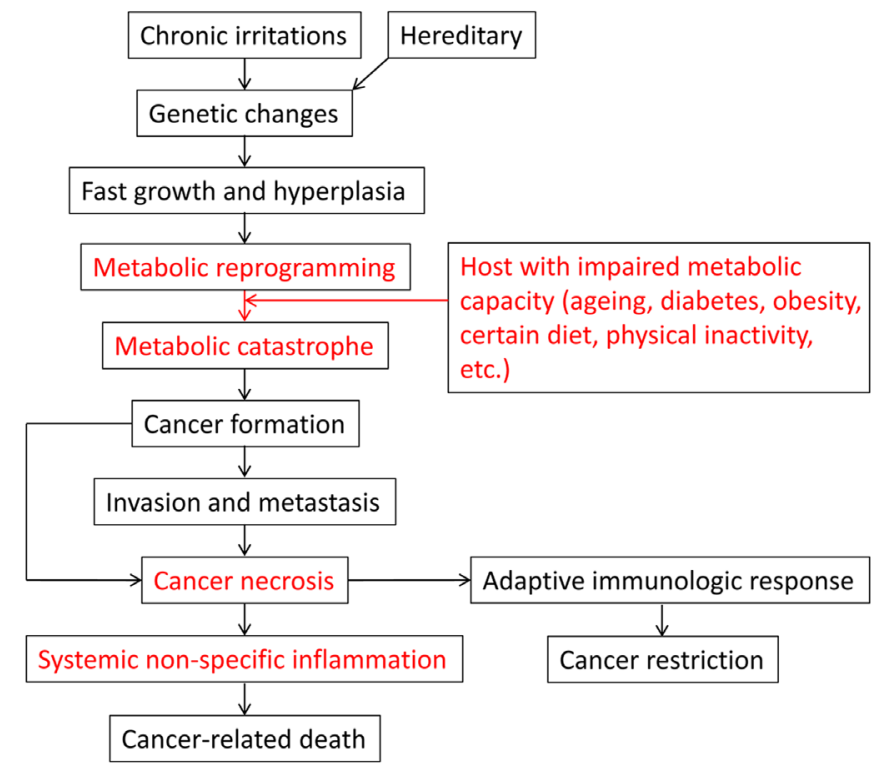

Figure 1. The integrative theory for cancer.

of O-GlcNAc synthesis promotes CA19-9 overproduction and cancer progression.

Obesity. Obesity has long been known as a risk factor of various types of cancer (53-55) and its prevalence has been rapidly increasing over the past two decades (55). Moreover, obesity is recognized as an adverse prognostic factor for cancer patients and it accounts for $14 \%$ of all cancer-related deaths in men and $20 \%$ in women in the US (56-58). Obese patients with cancer are often diagnosed at an advanced stage (55). The connection between obesity and cancer mainly relates to metabolic reprogramming caused by the prevalence of type II diabetes and insulin resistance, leading to insulin and IGF-1 overproduction $(55,59)$. Increased levels of circulating free fatty acids in obese individuals induce insulin resistance as a consequence of metabolic adaptation (55). In addition, chronic inflammation is common in obese individuals, which further promotes cancer evolvement (59). For example, in hepatocellular carcinoma, obesity enhances the production of the tumor-promoting cytokines, interleukin (IL)- 6 and tumor necrosis factor (TNF), leading to liver inflammation and activation of the oncogenic transcription factor, signal transducer and activator of transcription 3 (STAT3) (60). Furthermore, autophagy is deregulated in obese individuals, and the dysfunction of autophagy in the context of apoptosis resistance may cause tumor cell necrosis and inflammation (59). In brief, obesity facilitates cancer evolvement by metabolic reprogramming, inflammatory reaction and autophagy dysfunction.

Diet. Contrary to normal cells, the metabolism of cancer cells is reprogrammed to meet growth needs, requiring augmented calories and certain nutrients $(61,62)$. Caloric restriction, the consumption of fewer calories without inducing malnutrition, decreases the incidence of spontaneous cancers and inhibits cancer growth, although the effects are not uniform $(61,62)$. It can be achieved by a 10 to $50 \%$ decrease in caloric intake (63). Certain signals including insulin, IGF-1, phosphoinositide 3-kinase (PI3K), phosphatase and tensin homolog

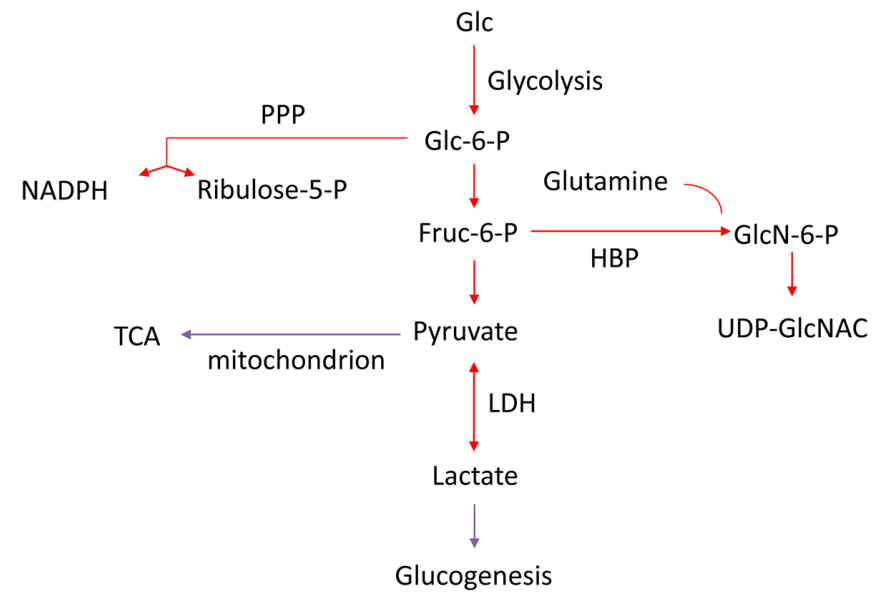

Figure 2. Cancer with a reprogrammed metabolism. The metabolism of cancer is reprogrammed to support its rapid growth, which strengthens the glycolysis, PPP, and HBP pathways but attenuates the TCA cycle and glucogenesis. PPP, pentose phosphate pathway; HBP, hexosamine biosynthetic pathway; TCA, tricarboxylic acid cycle; $\mathrm{LDH}$, lactate dehydrogenase.

(PTEN) and mammalian target of rapamycin (mTOR) are associated with the reaction of dietary restriction (63), which can affect the response of tumors cells to major treatments $(64,65)$. For example, tumor cells present different reactions to high-dose chemotherapy from normal cells under conditions of dietary restriction, which is potentially mediated by homolog Ras2val19 and IGF-1 $(64,65)$. Furthermore, certain amino acids, such as leucine $(66)$, glutamine $(67,68)$, serine (69), asparagine (70) and glycine (71) play an important role in cancer progression by interacting with major oncogenic signals, including KRAS, c-Myc and TP53. Therefore, caloric restriction and the restraint of certain nutrients may be promising strategies for cancer management.

\section{Metabolic imbalance induces cancer formation}

As mentioned above, the metabolism of tumor cells is rewired to support unrestricted growth with rapid energy supply and anabolic materials (6). Moreover, certain byproducts (lactate, non-essential amino acids, oncometabolites, etc.) are increased and thus tumors require detoxification mechanisms to maintain easy growth (8). During physiological activities, accumulated metabolites can be recycled or excreted through bodies. For example, lactate produced during sports can be reused by the lactic acid cycle through the gluconeogenesis pathway, or can be changed into pyruvate and then entered the TCA cycle (72). For solid cancers, the host not only provides 'fuel' for tumor growth, but also functions as the exhaust system via the tumor microenvironment. Angiogenesis, some tumor cells (reverse Warburg effect), and stromal cells in the tumor microenvironment are coupled with cancers to support tumor growth by providing nutrients, recycling oncometabolites and transporting metabolic wastes (17,73-76). Whether a metabolic balance between the tumor and host can be reached not only depends on the amount of oncometabolites produced by tumor cells, but more importantly, on the metabolic capacity of the host (Fig. 3A). Diabetes, obesity, aging, fatty liver, alcohol consumption, chronic site inflammation, physical inactivity and the intake of certain types of food can impair the 

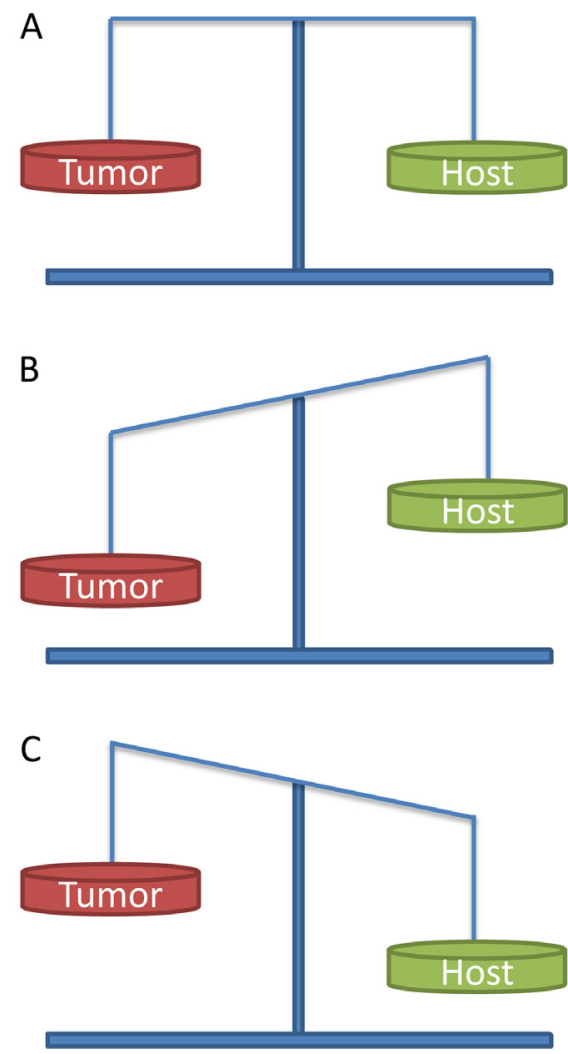

Figure 3. Metabolic interaction between tumor and host. (A) There is a metabolic balance in benign tumors and some indolent cancers. (B) For most adult patients with cancer, metabolic imbalance exists as the human body cannot sustain the needs of the rapidly growing tumor, leading to tumor cells becoming invasive and metastatic. (C) For young patients with sarcoma, metabolic imbalance occurs as the human body provides sufficient fuel and a powerful exhaust system to support its rapid growth.

metabolic capacity of the host, resulting in a metabolic imbalance between the tumor and host (Fig. 3B). The metabolic imbalance causes a metabolic catastrophe, compelling tumor cells to live in a harsh condition, which stimulates tumor death and provokes tumor cells to invade and metastasize. This is similar to a problematic car mismatched an advanced motor with an out-of-date exhaust system. Therefore, the evolvement of precancerous lesions (relative metabolic balance) to malignancy (moderate metabolic imbalance) and finally to metastatic cancer (severe metabolic imbalance) is driven by the metabolic imbalance between the tumor and host. For highly aggressive malignancies, the period from metabolic balance to imbalance is transient, making in situ carcinoma rapidly evolve into invasive cancer.

\section{Metabolic imbalance forces the tumor to invade and me- tastasize}

Although tumor cells can recruit stromal cells and promote angiogenesis to support their growth, the metabolic imbalance between the tumor and host can cause the accumulation of metabolic wastes, creating a dismal environment with metabolic catastrophe unsuited for tumor growth. The metabolic catastrophe stimulates tumor cells to invade adjacent tissues and metastasize to distant organs to couple their metabolic needs $(77,78)$. For instance, pancreatic cancer usually resides in a harsh environment with metabolic catastrophe (diabetes and chronic inflammation) and local invasion to nearby tissues (normal pancreas, nerves, bile duct and vessels) is common and associated with a poor prognosis (79). Moreover, the accumulation of metabolic waste can promote the death of tumor cells by necrosis, but not by apoptosis or autophagy (80). The inflammatory environment induced by necrosis further facilitates tumor invasion and metastasis $(81,82)$. Therefore, tumor invasion and metastasis are provoked by the metabolic imbalance between the tumor and host.

Metastasis occurs at an advanced stage of cancer evolvement. In 1889, Dr Stephen Paget proposed the well-known 'seed and soil' theory for cancer metastasis. However, even the sweetest soil is tougher than the soil in which the primary tumor resides (83). Metastasis is an inefficient process through which only a minority of migrating tumor cells can form metastatic foci $(84,85)$. In the primary tumor, stromal cells, certain tumor cells (reverse Warburg effect) and angiogenesis are coupled to re-use and excrete metabolic wastes (17). Although metastatic niches are preconditioned for tumor colonization $(86,87)$, different organs harbor distinct metabolic patterns and metastatic cancer cells that colonize different organs engage in different metabolic preferences (88). As regards breast cancer cells with a multiple metastatic capability (liver and lung), liver metastatic breast cancer cells use glucose uptake and glycolysis, while lung metastatic breast cancer cells employ glutamine uptake and oxidative phosphorylation (OXPHOS) to metastasize (89). Metastatic targeting is thus to do more with metabolic needs than simply a hemodynamic issue. In addition, as metastatic tumors can recruit less stromal cells and vasculature than the primary tumor, or tumor cells with the reverse Warburg effect may be incapable of metastasis, metastatic cells reside in a tough foreign microenvironment, causing a more severe metabolic catastrophe in metastases than that in the primary tumor $(88,90)$.

\section{Metabolic imbalance stimulates cachexia}

Cancer cachexia is defined as a multifactorial syndrome presenting an ongoing loss of skeletal muscle mass (with or without loss of adipose tissue) that can be partially, but not entirely reversed by conventional nutritional support and leads to progressive functional impairment (91). It is often considered a paraneoplastic syndrome which is characterized by weight loss and inflammation (92-94). It becomes evident at the final stage of cancer and predicts a poor survival (94). Cancer cachexia is induced by a negative energy balance caused by a decreased food intake and increased energy expenditure (92). When human bodies consume enough exogenous nutrients, tumors mainly consume exogenous nutrients to sustain their rapid growth. However, the intake of nutrients in cancer patients is often reduced due to symptoms, such as fever, anorexia, pain and chronic systemic inflammatory response (95). Moreover, the needs for nutrients are increased in cancers for their inefficient consumption of nutrients (Warburg effect) and a systemic inflammatory response by secreting pro-cachexia mediators, such as transforming growth factor $\beta$ (TGF $\beta$ ), prostaglandin E2 (PGE2), IL-6, IL-1, TNF and interferon $\gamma$ (IFN $\gamma)$ (92-94). The negative energy balance induces the degradation of skeletal muscle and fat mass, 
leading to a set of cachexia-related symptoms, including severe thinness, weakness, fatigue and anemia (92-95). Therefore, cachexia is a hint of a cancer-related metabolic disorder.

\section{Metabolic imbalance provokes systemic non-specific in- flammation via tumor necrosis}

There are three fundamental types of mammalian cell death, that is apoptosis, autophagy-associated cell death and necrosis (96). Apoptosis, a type of programmed cell death and an activating process by the consumption of ATP, is featured by the shrinkage of the cell and its nucleus without an obvious inflammatory response (97). Resistance to apoptosis is an essential feature of cancer development and effective chemotherapy and radiation act mainly through the induction apoptosis (98). Autophagy-associated cell death, a genetically controlled process, allows a starving or a sublethal cell to survive by recycling energy and metabolites via lysosome digesting their own organelles, particles and macromolecules (99). It is critical for maintaining cellular homeostasis and plays a context-dependent role in cancer evolvement (100). Necrosis, usually viewed as a non-programmed form of cell death, is caused by the rapid depletion of ATP (hypoxia, ischemia, or tumor growth outpacing angiogenesis), external stresses (infection, toxins, mechanical force, heat, or cold), and when apoptosis and autophagy are unlikely to continue if death stimuli persist $(96,97,101,102)$. In the process of necrosis, the early rupture of surface membranes allows an influx of extracellular fluids and a spillage of intracellular contents, resulting in cell and organelle swelling (96). It is worth mentioning that some types of necrosis are programmed, which are known as necroptosis, including TNF-induced programmed necrosis controlled by receptor-interacting serine/threonine-protein kinase 1 (RIP1) and receptor-interacting serine/threonine-protein kinase 3 (RIP3) (101). Unlike apoptosis and autophagy-associated cell death, necrosis is usually accompanied by an inflammatory response $(96,97,101,102)$.

The accumulation of metabolic wastes together with the lack of ATP supply lead to a metabolic catastrophe for cancer cells and ultimately cause their death (7). For cancer cells residing in a mild-stress environment, apoptosis is the main form of death for tumors with sufficient ATP supply (102). Furthermore, cancer cells require the catabolic process of autophagy to sustain an alternate energy source in periods of metabolic deprivation to prevent necrosis when the apoptosis of tumor cells is dysfunctional $(80,103)$. However, when metabolic catastrophe continues, or when the degree of metabolic stress becomes severe, metabolic stress triggers necrosis, which then becomes the major form of cancer cell death (80). Tumor necrosis is thus induced by metabolic catastrophe.

Coagulative necrosis is a common pathological feature of solid tumors (104-106). The presence of tumor necrosis has been reported as a dismal prognostic predictor in solid tumors (104-107). Following necrosis, tumor DNA is released outside the cell and becomes damage-associated molecular-pattern (DAMPs) (108). DAMPs, such as high mobility group box 1 (HMGB1) protein, can enter the circulation and activate a non-infectious inflammatory response (108). Tumor necrosis can induce both innate and adaptive immunologic responses, which are a 'double-edged sword' for cancer progression (109). Unlike apoptosis, in which several of the highly immunogenic intracellular proteins are sequestered into the dead cell, or autophagy-related cell death, in which the highly immunogenic intracellular proteins are degraded, necroptosis is a strong trigger of the adaptive immunologic responses, providing a critical and specific defense mechanism against cancer. Moreover, the detrimental side of necrosis is non-specific systemic inflammation, which can ultimately favor tumor progression and promote the death of the host (109).

\section{Systemic non-specific inflammation causes cancer-relat- ed mortality}

Mechanic destruction caused by the primary tumor and distant metastases is widely believed to be the cause of cancer-related mortality (110). Moreover, organ dysfunction caused by metastases is often deemed as the major reason (110-112). For example, hepatic dysfunction caused by hepatic metastases is generally deemed as the major reason of pancreatic cancer-related mortality (79). However, a study demonstrated that a large proportion $(63 \%)$ of patients with pancreatic cancer did not die of widespread metastatic disease, and even $12 \%$ of patients died of local pancreatic cancer (113). Another study found that the fraction (20\%) of cases with diffuse liver metastases was relatively small at the time of death and only a minor proportion (14\%) of patients with pancreatic cancer died from hepatic failure as a direct result of liver metastases (114). In fact, for healthy human beings, 1/3 of the normal liver volume can sustain the need of regular physiological activities. It is rarely observed that metastases of pancreatic cancer spread over $2 / 3$ of the liver volume (114). Briefly, organ dysfunction caused by metastases is not the major immediate reason of cancer-related mortality.

Cancer-associated inflammation is a pivotal component of tumor progression and a critical determinant of outcome in patients with cancer $(17,110,115)$. In early 1863 , Rudolf Virchow observed leucocytes in neoplastic tissues and made a connection between the sites of chronic inflammation and cancer. Recent data of the inflammatory reaction in the tumor microenvironment has supported Virchow's hypothesis (17). Inflammation is now included as a hallmark of cancer and reciprocal interactions between cancer and inflammation accelerate the neoplastic process $(17,81,116)$. To a certain degree, tumors are considered as wounds that never heal (116). Moreover, the links between cancer and inflammation are fostering novel anti-inflammatory therapeutic approaches for cancer prevention and treatment (81). However, previous studies have mainly focused on local inflammation surrounding the tumor, and have payed less attention to systemic inflammation in the host.

Cancer-related systemic inflammation, including hematopoietic changes (leucocytes, interleukins, interferons, hematopoietic growth factors, acute phase proteins, etc.) and alterations in neuroendocrine metabolism (endocrine hormones), has a more severe impact on the body than site inflammation in the tumor microenvironment (95). In general, for early-stage cancers, local inflammation is prominent at the primary tumor site; as tumor progression and systemic inflammation become more evident, inflammation peaks at the 
end stage of the disease, and induces a cachexic status (95). In ancient Greece, Hippocrates proposed the humoral theory, namely that the body had four humors: Black bile, yellow bile, blood and phlegm. He believed that an excess of black bile in various body sites caused cancer. This theory was embraced by the medical teachings of the influential doctor Galen. In the 17th century, the Dutch physician and chemist, Francois de la Boe Sylvius, an earliest defender of the theory of circulation of the blood, proposed that cancer was the outcome of chemical processes. Although the theory is debatable, inflammatory gradients in the blood system may play a similar role in cancer as 'black bile' in the humoral theory. Nowadays, the impact of cancer-related systemic inflammation upon the body has been well-established $(115,117,118)$. In a study on 506 patients with various types of cancer, the single major reason of death was infection (36\%) and it was also a contributory variable in an additional $68 \%$ of the patients, while organ invasion by neoplastic cells, including hepatic failure was the reason of death in $10 \%$ and a contributory factor in $5 \%$ of the cases (119). The degree of systemic inflammation can be effectively measured by the neutrophil-to-lymphocyte ratio (NLR), C-reactive protein (CRP) concentration and the Glasgow Prognostic Score (GPS), and these markers can be used to refine the stratification of patients to therapy and predict prognosis $(115,117,118)$. The computed tomography 'bull's-eye sign' of hepatic metastases suggests that tumor necrosis is more common in metastases than in the primary tumor, indicating that the systemic inflammation induced by metastases is more severe than that induced by the primary tumor. This could explain why $>90 \%$ of cancer-related mortality is attributable to metastases, while usually no obvious organ failure is found at the time of patient's death $(83,88,110,111)$. Therefore, systemic aseptic inflammation is the major immediate cause of cancer-related mortality. To a certain degree, the immune system is designed not only to protect, but also to terminate human beings.

\section{Hexosamine biosynthetic pathway (HBP) pathway regu- lates metabolism and cancer evolvement}

The HBP pathway, another branch of glycolysis apart from the PPP pathway, constitutes of approximately 1-3\% of total glucose flux in normal cells (120). It plays a prominent role in sensing nutrients fluctuation and regulating cellular metabolism through $\mathrm{O}$ - and $\mathrm{N}$-linked glycosylation of serine and threonine residues, the most abundant and structurally diverse post-translational modification $(121,122)$. UDP-GlcNAc, a sensing molecule of metabolism, is the end product of the HBP pathway and O-GlcNAc transferase (OGT) adds O-GlcNAc to target proteins, while O-GlcNAcase (OGA) removes the modification $(123,124)$. The HBP flux is governed by both nutrient availability and nutrient-processing enzymes (125). Glucose, glutamine and Acetyl-CoA are substrates of the HBP biosynthesis and can affect the entry of nutrients into the HBP pathway (125). The HBP flux has been involved in a wide range of chronic conditions, such as cancer, diabetes, neurodegenerative disease and aging $(36,123)$. In cancer cells, the HBP pathway is highly augmented, which can be clearly demonstrated by widely used carbohydrate related biomarkers [CA19-9, CEA, carbohydrate antigen 125 (CA125), etc.] $(121,124)$. Oncogenic signals, such as KRAS can increase the flux of the HBP pathway by upregulating key enzymes of HBP and increasing the levels of glucosamine-6-phosphate $(\mathrm{GlcN}-6 \mathrm{P})$, the product of the committed step governing flux into HBP (16). The augmented HBP pathway has a profound effect on cancer at various pathophysiological steps of tumor invasion and metastasis, immune evasion and the inflammatory response $(121,124,126)$. In addition, it also plays a fundamental role in the coordination of nutrient uptake and metabolism, partially by modulating glutamine uptake and interplaying with other nutrient sensor pathways, such as GH-IGF1, mTOR, SIRT1, PI3K-AKT and the energy sensor AMP-activated protein kinase (AMPK) $(21,126)$. Therefore, as branches of glycolysis, the PPP pathway sustains cancer growth, while the HBP pathway enables cancer cells to evolve. A diet rich in substrates of the HBP pathway may modulate the progression of cancer (68).

\section{The integrative theory applied in specific conditions}

For young patients with cancer, particularly sarcoma, metabolic imbalance exists as the host provides a powerful metabolic system for tumor growth. The metabolic capacity even outpaces the growth needs of tumors cells (Fig. 3C). Rapidly proliferating mesenchymal cells in children are vulnerable to various types of stresses, leading to malignant alterations with genetic mutations. Stromal cells create a nutrient-rich microenvironment to support tumor rapid growth and thus young patients are usually diagnosed with large tumors (75). Moreover, necrosis is not the major form of tumor death and it mostly presents in tumors with a low oxygen and blood supply, thus suggesting that these tumors are rarely associated with immune cell infiltration and inflammatory reaction (82).

For hematological malignancies, although the tumor microenvironment differs from that of solid tumors and metabolic wastes are recycled and excreted in a unique pattern, the metabolic and immunological capacity of the host also affects cancer evolvement.

For age-related death, although individuals are dead without tumor and other organic diseases, a systematic non-specific inflammation is also the major immediate reason. The systematic non-specific inflammation is induced by a metabolic imbalance caused by self-renewal needs and an impaired metabolism.

\section{Therapeutic potential}

It is well-recognized that cancer is exclusively a systemic disease with local appearance. Thus, tumor biology is not the properties of tumors, but the properties of an ecosystem containing both tumor and host. Modern therapeutic strategies are mainly focused on tumors, but rarely on the host. Researchers often note that experimental results are rarely repeated in patients during clinical trials. Experiments can be performed to examine the therapeutic efficacy by inhibiting uncontrolled tumor growth. However, as in vitro experiments have no external metabolism and the metabolic system of animal models differs from that of human beings, it is hard to replicate experimental results in patients with cancer during 


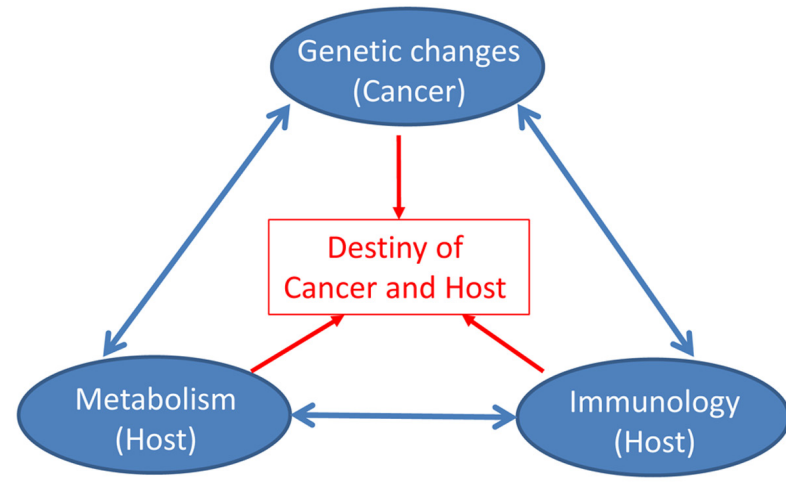

Figure 4. Diagram of the integrative theory for cancer. Three basic elements for cancer development in the integrative theory: genetic alterations (cancer), metabolic imbalance (host), and immunologic response (host). Interactions exist between these three factors. The combinedeffect of these elements determines the destiny of both cancer and host.

clinical trials. Therefore, therapeutic strategies should be an integrative mode by inhibiting uncontrolled tumor growth and improving host metabolic capacity. The metabolic capacity of human body can be impaired in certain circumstances, such as aging, diabetes, obesity, a high-fat and high-protein diet and physical inactivity. Accordingly, the correction of systemic metabolic disorders should be always kept in mind (calorie control, low-fat diet, proper exercise, controlling blood glucose levels, lose weight, metformin, etc.) (127). Furthermore, as systemic aseptic inflammation is the major immediate cause of cancer-related mortality, anti-inflammatory agents (e.g., non-steroidal anti-inflammatory drugs) could be used to control cancer progression (128). Activating adaptive immunologic response is a potential anti-cancer strategy, which is widely used in clinical practice nowadays (129).

For the fight against cancer, improving the metabolic and immunologic capacity of host should be as important as inhibiting cancer uncontrolled growth. Traditional Chinese medicine places great importance on improving the function of the human body. It emphasizes that human beings should live in harmony with nature. For cancers, it stresses cancer is not a disease, but rather a type of imbalance in the human body; thus, by improving the metabolic and immunologic capacity of the host, which may provide important clues for modern cancer treatment.

\section{Conclusion}

In this integrative mode, chronic irritations induce tumor with genetic changes and rapid proliferation. Tumor cells employ aerobic glycolysis to sustain uncontrolled rapid growth. The host provides both nutrients and the exhaust system to support tumor growth via the tumor microenvironment. Whether a metabolic balance between tumor and host can be reached depends not only on the amount of oncometabolites produced by tumor cells, but more importantly, on the metabolic capacity of the host. Under certain circumstances, such as aging, diabetes, obesity and a high-fat diet, the exhaust system is impaired, inducing a metabolic imbalance between the tumor and host. The metabolic imbalance causes a metabolic catastrophe, forcing tumor cells to undergo necrosis, and invade and metastasize. Tumor invasion and metastasis is thus a passive, but not an active process. Intact apoptosis or autophagy allow tumor cells to live with the host in a homeostasis. However, tumor necrosis, particularly in metastases, leads to a systemic non-specific inflammation, which is the major cause of cancer-related mortality. This could explain why the majority of cancer-related mortality is attributable to metastases even without obvious organ dysfunction. Moreover, chronic irritation (e.g., smoking) or genetic alterations (e.g., KRAS mutations) cannot induce cancer development in metabolically intact individuals. Cancer residing in a host with metabolic defects usually presents an aggressive feature. Therefore, cancer can somewhat be viewed as the most severe complication of metabolic disturbance. Therapeutic strategies should perhaps follow an integrative mode by inhibiting uncontrolled tumor growth and improving host metabolic capacity. Traditional Chinese medicine may provide important clues for modern cancer treatment by proposing that the host should live in 'harmony' with cancer; i.e, instead of fighting the cancer, rather restore the body's balance.

In brief, there are three basic elements of cancer development in the integrative theory: Genetic alterations (cancer), metabolic imbalance (host) and immunologic response (host) (Fig. 4). This theory views cancer in an integrative manner and proposes that both genetic changes and tumor-host interaction in metabolism and immunology determine the destiny of cancer and host. Although cancer is fundamentally a genetic disease, tumor biology is basically the nature of host.

\section{Acknowledgements}

Not applicable.

\section{Funding}

This study was funded by the National Natural Science Foundation of China (no. 81871940), the Shanghai Cancer Center Foundation for Distinguished Young Scholars (no. YJJQ201803), and the Shanghai Natural Science Foundation (no. 17ZR1406300).

\section{Availability of data and materials}

Not applicable.

\section{Authors' contributions}

GL and NL conceived the review and analyzed the relevant literature. GL and NL sourced the literature and wrote the first draft of the manuscript. GL and NL critically revised the manuscript. GL produced the figures. Both authors have read and approved the final manuscript.

\section{Ethics approval and consent to participate}

Not applicable.

\section{Patient consent for publication}

Not applicable. 


\section{Competing interests}

The authors confirm that they have no competing interests.

\section{References}

1. Bray F, Ferlay J, Soerjomataram I, Siegel RL, Torre LA and Jemal A: Global cancer statistics 2018: GLOBOCAN estimates of incidence and mortality worldwide for 36 cancers in 185 countries. CA Cancer J Clin 68: 394-424, 2018.

2. Torre LA, Bray F, Siegel RL, Ferlay J, Lortet-Tieulent J and Jemal A Global cancer statistics, 2012. CA Cancer J Clin 65: 87-108, 2015.

3. Vineis P and Wild CP: Global cancer patterns: Causes and prevention. Lancet 383: 549-557, 2014.

4. Soerjomataram I, Lortet-Tieulent J, Parkin DM, Ferlay J, Mathers C, Forman D and Bray F: Global burden of cancer in 2008: A systematic analysis of disability-adjusted life-years in 12 world regions. Lancet 380: 1840-1850, 2012.

5. Warburg O: On respiratory impairment in cancer cells Science 124: 269-270, 1956.

6. Jin S, DiPaola RS, Mathew R and White E: Metabolic catastrophe as a means to cancer cell death. J Cell Sci 120: 379-383, 2007.

7. Gatenby RA and Gillies RJ: Why do cancers have high aerobic glycolysis? Nat Rev Cancer 4: 891-899, 2004.

8. Hsu PP and Sabatini DM: Cancer cell metabolism: Warburg and beyond. Cell 134: 703-707, 2008.

9. Koppenol WH, Bounds PL and Dang CV: Otto Warburg's contributions to current concepts of cancer metabolism. Nat Rev Cancer 11: 325-337, 2011.

10. Vander Heiden MG, Cantley LC and Thompson CB: Understanding the Warburg effect: The metabolic requirements of cell proliferation. Science 324: 1029-1033, 2009.

11. Vander Heiden MG and DeBerardinis RJ: Understanding the Intersections between Metabolism and Cancer Biology. Cell 168: 657-669, 2017

12. Casás-Selves M and Degregori J: How cancer shapes evolution, and how evolution shapes cancer. Evolution (NY) 4: 624-634, 2011

13. Bishop JM: Molecular themes in oncogenesis. Cell 64: 235-248, 1991.

14. Spandidos DA and Wilkie NM: Malignant transformation of early passage rodent cells by a single mutated human oncogene. Nature 310: 469-475, 1984.

15. Spandidos DA: A unified theory for the development of cancer. Biosci Rep 6: 691-708, 1986.

16. Ying H, Kimmelman AC, Lyssiotis CA, Hua S, Chu GC, FletcherSananikone E, Locasale JW, Son J, Zhang H, Coloff JL, et al: Oncogenic Kras maintains pancreatic tumors through regulation of anabolic glucose metabolism. Cell 149: 656-670, 2012.

17. Hanahan D and Weinberg RA: Hallmarks of cancer: The next generation. Cell 144: 646-674, 2011

18. Moussaieff A, Rouleau M, Kitsberg D, Cohen M, Levy G, Barasch D, Nemirovski A, Shen-Orr S, Laevsky I, Amit M, et al: Glycolysis-mediated changes in acetyl-CoA and histone acetylation control the early differentiation of embryonic stem cells. Cell Metab 21: 392-402, 2015

19. Hammarström S: The carcinoembryonic antigen (CEA) family: Structures, suggested functions and expression in normal and malignant tissues. Semin Cancer Biol 9: 67-81, 1999.

20. Richardson LC and Pollack LA: Therapy insight: Influence of type 2 diabetes on the development, treatment and outcomes of cancer. Nat Clin Pract Oncol 2: 48-53, 2005.

21. Wellen KE,Lu C, Mancuso A,Lemons JM, Ryczko M, Dennis JW, Rabinowitz JD, Coller HA and Thompson CB: The hexosamine biosynthetic pathway couples growth factor-induced glutamine uptake to glucose metabolism. Genes Dev 24: 2784-2799, 2010.

22. Wellen KE and Thompson CB: Cellular metabolic stress: Considering how cells respond to nutrient excess. Mol Cell 40: 323-332, 2010.

23. Joslin EP, Lombard HL, Burrows RE and Manning MD: Diabetes and cancer. N Engl J Med 260: 486-488, 1959.

24. Pearson-Stuttard J, Zhou B, Kontis V, Bentham J, Gunter MJ and Ezzati M: Worldwide burden of cancer attributable to diabetes and high body-mass index: A comparative risk assessment Lancet Diabetes Endocrinol 6: 95-104, 2018.

25. Yuan C, Rubinson DA, Qian ZR, Wu C, Kraft P, Bao Y, Ogino S, $\mathrm{Ng} \mathrm{K}$, Clancy TE, Swanson RS, et al: Survival among patients with pancreatic cancer and long-standing or recent-onset diabetes mellitus. J Clin Oncol 33: 29-35, 2015.
26. Niccoli T and Partridge L: Ageing as a risk factor for disease. Curr Biol 22: R741-R752, 2012.

27. Jemal A, Bray F, Center MM, Ferlay J, Ward E and Forman D: Global cancer statistics. CA Cancer J Clin 61: 69-90, 2011.

28. Campisi J: Aging, cellular senescence, and cancer. Annu Rev Physiol 75: 685-705, 2013.

29. Hinkal G, Parikh N and Donehower LA: Timed somatic deletion of p53 in mice reveals age-associated differences in tumor progression. PLoS One 4: e6654, 2009.

30. DePinho RA: The age of cancer. Nature 408: 248-254, 2000.

31. Stanta G, Campagner L, Cavallieri F and Giarelli L: Cancer of the oldest old. What we have learned from autopsy studies. Clin Geriatr Med 13: 55-68, 1997.

32. Dollé ME, Snyder WK, Gossen JA, Lohman PH and Vijg J: Distinct spectra of somatic mutations accumulated with age in mouse heart and small intestine. Proc Natl Acad Sci USA 97: 8403-8408, 2000.

33. Dollé ME, Giese H, Hopkins CL, Martus HJ, Hausdorff JM and Vijg J: Rapid accumulation of genome rearrangements in liver but not in brain of old mice. Nat Genet 17: 431-434, 1997.

34. Miller RA: The aging immune system: Primer and prospectus. Science 273: 70-74, 1996.

35. Bright R. Cases and observations connected with disease of thepancreas and duodenum. Med Chir Trans. 1833;18:1Y56.

36. Everhart J and Wright D: Diabetes mellitus as a risk factor for pancreatic cancer. A meta-analysis. JAMA 273: 1605-1609, 1995

37. Larsson SC, Orsini N and Wolk A: Diabetes mellitus and risk of colorectal cancer: A meta-analysis. J Natl Cancer Inst 97: $1679-1687,2005$

38. Bragg F, Holmes MV, Iona A, Guo Y, Du H, Chen Y, Bian Z, Yang L, Herrington W, Bennett D, et al; China Kadoorie Biobank Collaborative Group: Association between diabetes and causespecific mortality in rural and urban areas of China. JAMA 317: 280-289, 2017.

39. Toriola AT, Stolzenberg-Solomon R, Dalidowitz L, Linehan D and Colditz G: Diabetes and pancreatic cancer survival: A prospective cohort-based study. Br J Cancer 111: 181-185, 2014.

40. Kleeff J, Costello E, Jackson R, Halloran C, Greenhalf W, Ghaneh P, Lamb RF, Lerch MM, Mayerle J, Palmer D, et al: The impact of diabetes mellitus on survival following resection and adjuvant chemotherapy for pancreatic cancer. Br J Cancer 115: 887-894, 2016.

41. Barone BB, Yeh HC, Snyder CF, Peairs KS, Stein KB, Derr RL, Wolff AC and Brancati FL: Long-term all-cause mortality in cancer patients with preexisting diabetes mellitus: A systematic review and meta-analysis. JAMA 300: 2754-2764, 2008

42. Cui Y and Andersen DK: Diabetes and pancreatic cancer. Endocr Relat Cancer 19: F9-F26, 2012.

43. Pollak M: Insulin and insulin-like growth factor signalling in neoplasia. Nat Rev Cancer 8: 915-928, 2008.

44. Sah RP, Nagpal SJ, Mukhopadhyay D and Chari ST: New insights into pancreatic cancer-induced paraneoplastic diabetes. Nat Rev Gastroenterol Hepatol 10: 423-433, 2013.

45. Slawson C, Copeland RJ and Hart GW: O-GlcNAc signaling: A metabolic link between diabetes and cancer? Trends Biochem Sci 35: 547-555, 2010.

46. Pannala R, Leirness JB, Bamlet WR, Basu A, Petersen GM and Chari ST: Prevalence and clinical profile of pancreatic cancerassociated diabetes mellitus. Gastroenterology 134: 981-987, 2008.

47. Pannala R, Leibson CL, Rabe KG, Timmons LJ, Ransom J, de Andrade M, Petersen GM and Chari ST: Temporal association of changes in fasting blood glucose and body mass index with diagnosis of pancreatic cancer. Am J Gastroenterol 104: 2318-2325, 2009.

48. Pannala R, Basu A, Petersen GM and Chari ST: New-onset diabetes: A potential clue to the early diagnosis of pancreatic cancer. Lancet Oncol 10: 88-95, 2009.

49. Luo G, Liu C, Guo M, Cheng H, Lu Y, Jin K, Liu L, Long J, Xu J, Lu R, et al: Potential biomarkers in Lewis negative patients with pancreatic cancer. Ann Surg 265: 800-805, 2017.

50. Luo G, Liu C, Guo M, Long J, Liu Z, Xiao Z, Jin K, Cheng $H$ Lu Y, Ni Q, et al: CA19-9-Low\&Lewis (+) pancreatic cancer: A unique subtype. Cancer Lett 385: 46-50, 2017.

51. Esteghamati A, Hafezi-Nejad N, Zandieh A, Sheikhbahaei S, Emamzadeh-Fard S and Nakhjavani M: CA 19-9 is associated with poor glycemic control in diabetic patients: Role of insulin resistance. Clin Lab 60: 441-447, 2014.

52. Kim SH, Baek CO, Lee KA, Park TS, Baek HS and Jin HY: Clinical implication of elevated CA 19-9 level and the relationship with glucose control state in patients with type 2 diabetes. Endocrine 46: 249-255, 2014. 
53. McWilliams RR and Petersen GM: Overweight, obesity, and pancreatic cancer: Beyond risk alone. JAMA 301: 2592-2593, 2009.

54. Bianchini F, Kaaks R and Vainio H: Overweight, obesity, and cancer risk. Lancet Oncol 3: 565-574, 2002.

55. Calle EE and Kaaks R: Overweight, obesity and cancer: Epidemiological evidence and proposed mechanisms. Nat Rev Cancer 4: 579-591, 2004.

56. Yuan C, Bao Y, Wu C, Kraft P, Ogino S, Ng K, Qian ZR, Rubinson DA, Stampfer MJ, Giovannucci EL, et al: Prediagnostic body mass index and pancreatic cancer survival. J Clin Oncol 31: 4229-4234, 2013.

57. Calle EE, Rodriguez C, Walker-Thurmond $\mathrm{K}$ and Thun MJ: Overweight, obesity, and mortality from cancer in a prospectively studied cohort of U.S. adults. N Engl J Med 348: 1625-1638, 2003

58. Hu FB, Willett WC, Li T, Stampfer MJ, Colditz GA and Manson JE: Adiposity as compared with physical activity in predicting mortality among women. N Engl J Med 351: 2694-2703, 2004.

59. Gukovsky I, Li N, Todoric J, Gukovskaya A and Karin M: Inflammation, autophagy, and obesity: Common features in the pathogenesis of pancreatitis and pancreatic cancer. Gastroenterology 144: 1199-1209.e1194, 2013.

60. Park EJ, Lee JH, Yu GY, He G, Ali SR, Holzer RG, Osterreicher $\mathrm{CH}$, Takahashi $\mathrm{H}$ and Karin M: Dietary and genetic obesity promote liver inflammation and tumorigenesis by enhancing IL-6 and TNF expression. Cell 140: 197-208, 2010.

61. Weindruch R and Walford RL: Dietary restriction in mice beginning at 1 year of age: Effect on life-span and spontaneous cancer incidence. Science 215: 1415-1418, 1982.

62. Dhahbi JM, Kim HJ, Mote PL, Beaver RJ and Spindler SR: Temporal linkage between the phenotypic and genomic responses to caloric restriction. Proc Natl Acad Sci USA 101: 5524-5529, 2004

63. Kalaany NY and Sabatini DM: Tumours with PI3K activation are resistant to dietary restriction. Nature 458: 725-731, 2009.

64. Lee C, Safdie FM, Raffaghello L, Wei M, Madia F, Parrella E, Hwang D, Cohen P, Bianchi G and Longo VD: Reduced levels of IGF-I mediate differential protection of normal and cancer cells in response to fasting and improve chemotherapeutic index. Cancer Res 70: 1564-1572, 2010

65. Raffaghello L, Lee C, Safdie FM, Wei M, Madia F, Bianchi G and Longo VD: Starvation-dependent differential stress resistance protects normal but not cancer cells against high-dose chemotherapy. Proc Natl Acad Sci USA 105: 8215-8220, 2008.

66. Sheen JH, Zoncu R, Kim D and Sabatini DM: Defective regulation of autophagy upon leucine deprivation reveals a targetable liability of human melanoma cells in vitro and in vivo. Cancer Cell 19: 613-628, 2011.

67. Gao P, Tchernyshyov I, Chang TC, Lee YS, Kita K, Ochi T, Zeller KI, De Marzo AM, Van Eyk JE, Mendell JT, et al: c-Myc suppression of $\mathrm{miR}-23 \mathrm{a} / \mathrm{b}$ enhances mitochondrial glutaminase expression and glutamine metabolism. Nature 458: 762-765, 2009.

68. Son J, Lyssiotis CA, Ying H, Wang X, Hua S, Ligorio M Perera RM, Ferrone CR, Mullarky E, Shyh-Chang N, et al: Glutamine supports pancreatic cancer growth through a KRASregulated metabolic pathway. Nature 496: 101-105, 2013.

69. Maddocks OD, Berkers CR, Mason SM, Zheng L, Blyth K, Gottlieb E and Vousden KH: Serine starvation induces stress and p53-dependent metabolic remodelling in cancer cells. Nature 493: 542-546, 2013.

70. Knott SRV, Wagenblast E, Khan S, Kim SY, Soto M, Wagner M, Turgeon MO, Fish L, Erard N, Gable AL, et al: Asparagine bioavailability governs metastasis in a model of breast cancer. Nature 554: 378-381, 2018.

71. Jain M, Nilsson R, Sharma S, Madhusudhan N, Kitami T, Souza AL, Kafri R, Kirschner MW, Clish CB and Mootha VK: Metabolite profiling identifies a key role for glycine in rapid cancer cell proliferation. Science 336: 1040-1044, 2012.

72. Gladden LB: Lactate metabolism: A new paradigm for the third millennium. J Physiol 558: 5-30, 2004.

73. Feron O: Pyruvate into lactate and back: From the Warburg effect to symbiotic energy fuel exchange in cancer cells. Radiother Oncol 92: 329-333, 2009

74. Sonveaux P, Végran F, Schroeder T, Wergin MC, Verrax J, Rabbani ZN, De Saedeleer CJ, Kennedy KM, Diepart C, Jordan BF, et al: Targeting lactate-fueled respiration selectively kills hypoxic tumor cells in mice. J Clin Invest 118: 3930-3942, 2008.

75. Martinez-Outschoorn UE, Lisanti MP and Sotgia F: Catabolic cancer-associated fibroblasts transfer energy and biomass to anabolic cancer cells, fueling tumor growth. Semin Cancer Biol 25: 47-60, 2014.
76. Goodwin ML, Jin H, Straessler K, Smith-Fry K, Zhu JF, Monument MJ, Grossmann A, Randall RL, Capecchi MR and Jones KB: Modeling alveolar soft part sarcomagenesis in the mouse: A role for lactate in the tumor microenvironment. Cancer Cell 26: 851-862, 2014

77. Doherty JR and Cleveland JL: Targeting lactate metabolism for cancer therapeutics. J Clin Invest 123: 3685-3692, 2013.

78. Shime H, Yabu M, Akazawa T, Kodama K, Matsumoto M, Seya T and Inoue N: Tumor-secreted lactic acid promotes IL-23/ IL-17 proinflammatory pathway. J Immunol 180: 7175-7183, 2008 .

79. Ryan DP, Hong TS and Bardeesy N: Pancreatic adenocarcinoma. N Engl J Med 371: 1039-1049, 2014.

80.Zong WX and Thompson CB: Necrotic death as a cell fate. Genes Dev 20: 1-15, 2006

81. Balkwill $\mathrm{F}$ and Mantovani A: Inflammation and cancer: Back to Virchow? Lancet 357: 539-545, 2001

82. Vakkila J and Lotze MT: Inflammation and necrosis promote tumour growth. Nat Rev Immunol 4: 641-648, 2004.

83. Vanharanta $\mathrm{S}$ and Massagué J: Origins of metastatic traits Cancer Cell 24: 410-421, 2013

84. Oskarsson T, Batlle E and Massagué J: Metastatic stem cells: Sources, niches, and vital pathways. Cell Stem Cell 14: 306-321, 2014.

85. Fidler IJ: The pathogenesis of cancer metastasis: The 'seed and soil' hypothesis revisited. Nat Rev Cancer 3: 453-458, 2003.

86. Peinado H, Zhang H, Matei IR, Costa-Silva B, Hoshino A, Rodrigues G, Psaila B, Kaplan RN, Bromberg JF, Kang Y, et al: Pre-metastatic niches: Organ-specific homes for metastases. Nat Rev Cancer 17: 302-317, 2017.

87. Fong MY, Zhou W, Liu L, Alontaga AY, Chandra M, Ashby J, Chow A, O'ConnorST,LiS, Chin AR, et al:Breast-cancer-secreted miR-122 reprograms glucose metabolism in premetastatic niche to promote metastasis. Nat Cell Biol 17: 183-194, 2015.

88. Pascual G, Domínguez D and Benitah SA: The contributions of cancer cell metabolism to metastasis. Dis Model Mech 11: 11, 2018

89. Dupuy F, Tabariès S, Andrzejewski S, Dong Z, Blagih J, Annis MG, Omeroglu A, Gao D, Leung S, Amir E, et al: PDK1-dependent metabolic reprogramming dictates metastatic potential in breast cancer. Cell Metab 22: 577-589, 2015.

90. Chambers AF, Groom AC and MacDonald IC: Dissemination and growth of cancer cells in metastatic sites. Nat Rev Cancer 2: 563-572, 2002.

91. Fearon K, Strasser F, Anker SD, Bosaeus I, Bruera E, Fainsinger RL, Jatoi A, Loprinzi C, MacDonald N, Mantovani G, et al: Definition and classification of cancer cachexia: An international consensus. Lancet Oncol 12: 489-495, 2011.

92. Baracos VE, Martin L, Korc M, Guttridge DC and Fearon KC Cancer-associated cachexia. Nat Rev Dis Primers 4: 17105, 2018

93. Argilés JM, Busquets S, Stemmler B and López-Soriano FJ Cancer cachexia: Understanding the molecular basis. Nat Rev Cancer 14: 754-762, 2014.

94. Fearon KC, Glass DJ and Guttridge DC: Cancer cachexia: Mediators, signaling, and metabolic pathways. Cell Metab 16 153-166, 2012

95. McMillan DC: Systemic inflammation, nutritional status and survival in patients with cancer. Curr Opin Clin Nutr Metab Care 12: 223-226, 2009.

96. Hotchkiss RS, Strasser A, McDunn JE and Swanson PE: Cell death. N Engl J Med 361: 1570-1583, 2009.

97. Danial NN and Korsmeyer SJ: Cell death: Critical control points. Cell 116: 205-219, 2004

98. Igney FH and Krammer PH: Death and anti-death: Tumour resistance to apoptosis. Nat Rev Cancer 2: 277-288, 2002.

99. Kroemer G and Jäättelä M: Lysosomes and autophagy in cell death control. Nat Rev Cancer 5: 886-897, 2005.

100. White E, Mehnert JM and Chan CS: Autophagy, Metabolism, and Cancer. Clin Cancer Res 21: 5037-5046, 2015.

101. Li J, McQuade T, Siemer AB, Napetschnig J, Moriwaki K, Hsiao YS, Damko E, Moquin D, Walz T, McDermott A, et al: The RIP1/RIP3 necrosome forms a functional amyloid signaling complex required for programmed necrosis. Cell 150: 339-350, 2012 .

102. Majno G and Joris I: Apoptosis, oncosis, and necrosis. An overview of cell death. Am J Pathol 146: 3-15, 1995

103. Degenhardt K, Mathew R, Beaudoin B, Bray K, Anderson D, Chen G, Mukherjee C, Shi Y, Gélinas C, Fan Y, et al: Autophagy promotes tumor cell survival and restricts necrosis, inflammation, and tumorigenesis. Cancer Cell 10: 51-64, 2006. 
104.Edwards JG, Swinson DE, Jones JL, Muller S, Waller DA and O'Byrne KJ: Tumor necrosis correlates with angiogenesis and is a predictor of poor prognosis in malignant mesothelioma. Chest 124: 1916-1923, 2003

105.Lee SE, Byun SS, Oh JK, Lee SC, Chang IH, Choe G and Hong SK: Significance of macroscopic tumor necrosis as a prognostic indicator for renal cell carcinoma. J Urol 176: 1332-1337, discussion 1337-1338, 2006.

106. Tomes L, Emberley E, Niu Y, Troup S, Pastorek J, Strange K, Harris A and Watson PH: Necrosis and hypoxia in invasive breast carcinoma. Breast Cancer Res Treat 81: 61-69, 2003.

107. Richards CH, Mohammed Z, Qayyum T, Horgan PG and McMillan DC: The prognostic value of histological tumor necrosis in solid organ malignant disease: A systematic review. Future Oncol 7: 1223-1235, 2011.

108. Lotze MT and Tracey KJ: High-mobility group box 1 protein (HMGB1): Nuclear weapon in the immune arsenal. Nat Rev Immunol 5: 331-342, 2005.

109. Kaczmarek A, Vandenabeele P and Krysko DV: Necroptosis: The release of damage-associated molecular patterns and its physiological relevance. Immunity 38: 209-223, 2013.

110. Gupta GP and Massagué J: Cancer metastasis: Building a framework. Cell 127: 679-695, 2006.

111. Valastyan S and Weinberg RA: Tumor metastasis: Molecular insights and evolving paradigms. Cell 147: 275-292, 2011.

112. Steeg PS: Tumor metastasis: Mechanistic insights and clinical challenges. Nat Med 12: 895-904, 2006.

113. Iacobuzio-Donahue CA, Fu B, Yachida S, Luo M, Abe H, Henderson CM, Vilardell F, Wang Z, Keller JW, Banerjee P, et al: DPC4 gene status of the primary carcinoma correlates with patterns of failure in patients with pancreatic cancer. J Clin Oncol 27: 1806-1813, 2009.

114. Nakahashi C, Oda T, Kinoshita T, Ueda T, Konishi M, Nakagohri T, Inoue K, Furuse J, Ochiai A and Ohkohchi N: The impact of liver metastasis on mortality in patients initially diagnosed with locally advanced or resectable pancreatic cancer. Int J Gastrointest Cancer 33: 155-164, 2003.

115. Guthrie GJ, Charles KA, Roxburgh CS, Horgan PG McMillan DC and Clarke SJ: The systemic inflammation-based neutrophil-lymphocyte ratio: Experience in patients with cancer. Crit Rev Oncol Hematol 88: 218-230, 2013.

116. Dvorak HF: Tumors: Wounds that do not heal. Similarities between tumor stroma generation and wound healing. N Engl J Med 315: 1650-1659, 1986.

117. Ishizuka M, Nagata $H$, Takagi $K$, Horie $T$ and Kubota $K$ : Inflammation-based prognostic score is a novel predictor of postoperative outcome in patients with colorectal cancer. Ann Surg 246: 1047-1051, 2007.
118. Luo G, Guo M, Liu Z, Xiao Z, Jin K, Long J, Liu L, Liu C, Xu J, $\mathrm{Ni}$ Q, et al: Blood neutrophil-lymphocyte ratio predicts survival in patients with advanced pancreatic cancer treated with chemotherapy. Ann Surg Oncol 22: 670-676, 2015.

119. Ambrus JL, Ambrus CM, Mink IB and Pickren JW: Causes of death in cancer patients. J Med 6: 61-64, 1975.

120. Marshall S, Bacote V and Traxinger RR: Discovery of a metabolic pathway mediating glucose-induced desensitization of the glucose transport system. Role of hexosamine biosynthesis in the induction of insulin resistance. J Biol Chem 266: 4706-4712, 1991.

121. Hart GW and Copeland RJ: Glycomics hits the big time. Cell 143: 672-676, 2010.

122. Wang J, Liu R, Hawkins M, Barzilai N and Rossetti L: A nutrient-sensing pathway regulates leptin gene expression in muscle and fat. Nature 393: 684-688, 1998

123. Bond MR and Hanover JA: O-GlcNAc cycling: A link between metabolism and chronic disease. Annu Rev Nutr 33: 205-229, 2013.

124. Fuster MM and Esko JD: The sweet and sour of cancer: Glycans as novel therapeutic targets. Nat Rev Cancer 5: 526-542, 2005.

125. Chiaradonna F, Ricciardiello $\mathrm{F}$ and Palorini R: The nutrient-sensing hexosamine biosynthetic pathway as the hub of cancer metabolic rewiring. Cells 7: 7, 2018

126. Bullen JW, Balsbaugh JL, Chanda D, Shabanowitz J, Hunt DF, Neumann D and Hart GW: Cross-talk between two essential nutrient-sensitive enzymes: O-GlcNAc transferase (OGT) and AMP-activated protein kinase (AMPK). J Biol Chem 289: 10592-10606, 2014

127. Pascual G, Avgustinova A, Mejetta S, Martín M, Castellanos A, Attolini CS, Berenguer A, Prats N, Toll A, Hueto JA, et al: Targeting metastasis-initiating cells through the fatty acid receptor CD36. Nature 541: 41-45, 2017.

128. Shin YK, Park JS, Kim HS, Jun HJ, Kim GE, Suh CO, Yun YS and Pyo H: Radiosensitivity enhancement by celecoxib, a cyclooxygenase (COX)-2 selective inhibitor, via COX-2-dependent cell cycle regulation on human cancer cells expressing differential COX-2 levels. Cancer Res 65: 9501-9509, 2005.

129. Topalian SL, Taube JM, Anders RA and Pardoll DM: Mechanism-driven biomarkers to guide immune checkpoint blockade in cancer therapy. Nat Rev Cancer 16: 275-287, 2016.

This work is licensed under a Creative Commons Attribution-NonCommercial-NoDerivatives 4.0 International (CC BY-NC-ND 4.0) License. 